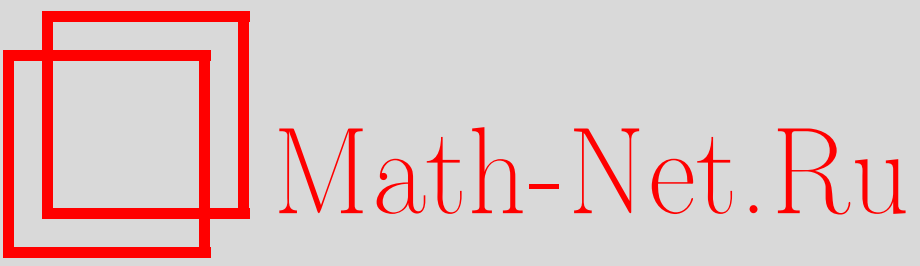

П. С. Геворкян, Эквивариантные расслоения, Итоги науки и техн. Сер. Соврем. мат. и ее прил. Темат. обз., 2020, том 180, 23-30

DOI: https://doi.org/10.36535/0233-6723-2020-180-23-30

Использование Общероссийского математического портала Math-Net.Ru подразумевает, что вы прочитали и согласны с пользовательским соглашением

http://www.mathnet.ru/rus/agreement

Параметры загрузки:

IP: 35.174 .16 .151

26 апреля 2023 г., 09:18:01 


\title{
ЭКВИВАРИАНТНЫЕ РАССЛОЕНИЯ
}

\author{
(C) 2020 г. П. С. ГЕВОРКЯН
}

\begin{abstract}
АннотАция. В работе изучаются эквивариантные расслоения Гуревича. Получены их внутренние характеристики. Доказаны теоремы о связях эквивариантных расслоений с порожденными ими расслоениями. Исследуются локальные и глобальные свойства эквивариантных расслоений. Доказан эквивариантный аналог теоремы Гуревича о переходе от локальных расслоений к глобальным расслоениям. Дана классификация эквивариантных расслоений со свойством единственности накрывающего пути.
\end{abstract}

Ключевъе слова: эквивариантная накрывающая гомотопия, эквивариантные расслоения, эквивариантная гомотопия, $H$-неподвижная точка, пространство $H$-орбит, нормальное $G$-покрытие, слабо локально тривиальное $G$-расслоение.

\section{EQUIVARIANT BUNDLES}

\section{(c) 2020 P. S. GEVORGYAN}

\begin{abstract}
In this paper, we study equivariant Hurewicz bundles, obtain their internal characteristics, and prove theorems on relationship between equivariant bundles and bundles generated by them. Local and global properties of equivariant bundles are examined. An equivariant analog of the Hurewicz theorem on passing from local bundles to global bundles is proved. A classification of equivariant bundles with the uniqueness property of the covering path is given.
\end{abstract}

Keywords and phrases: equivariant covering homotopy, equivariant bundles, equivariant homotopy, $H$-fixed point, $H$-orbit space, normal $G$-covering, weakly locally trivial $G$-bundle.

AMS Subject Classification: 55R05, 55R65, 55R91

1. Введение. Пусть $G$-топологическая группа, $E, B$ и $X$-произвольные $G$-пространства, a $p: E \rightarrow B$ и $f: X \rightarrow B$ - некоторые эквивариантные отображения. Задача поднятия эквивариантного отображения $f$ в $E$ состоит в нахождении такого эквивариантного отображения $\tilde{f}: X \rightarrow E$, что $f=p \circ \tilde{f}$. Для того чтобы эта задача стала корректной задачей $G$-гомотопической категории, отображение $p: E \rightarrow B$ должно удовлетворять дополнительному условию.

Говорят, что эквивариантное отображение $p: E \rightarrow B$ обладает свойством эквивариантной накрывающей гомотопии относительно $G$-пространства $X$, если для произвольных эквивариантных отображений $\tilde{f}: X \rightarrow E$ и $F: X \times I \rightarrow B$ таких, что $F \circ i_{0}=p \circ \tilde{f}$, где $i_{0}: X \rightarrow X \times I-$ вложение, определенное формулой $i_{0}(x)=(x, 0), x \in X$, существует такое эквивариантное отображение $\tilde{F}: X \times I \rightarrow E$, что коммутативна диаграмма

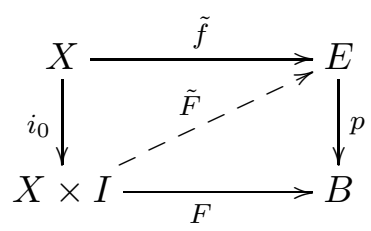

т.е. $p \circ \tilde{F}=F$ и $\tilde{F} \circ i_{0}=\tilde{f}$. 
Если $p: E \rightarrow B$ обладает свойством эквивариантной накрывающей гомотопии относительно $G$-пространства $X$, а $f, g: X \rightarrow B-G$-гомотопные эквивариантные отображения, то $f$ можно поднять в $E$ тогда и только тогда, когда $g$ можно поднять в $E$. Иначе говоря, поднятие эквивариантного отображения $f: X \rightarrow B$ является свойством $G$-гомотопического класса этого отображения.

Свойство эквивариантной накрывающей гомотопии приводит к понятию эквивариантного расслоения. Эквивариантное отображение $p: E \rightarrow B$ называется эквивариантным расслоением Гуревича или $G$-расслоением Гуревича, если $p$ обладает свойством эквивариантной накрывающей гомотопии относительно любого $G$-пространства $X$.

В данной статье изучаются эквивариантные расслоения Гуревича. Получены их внутренние характеристики с помощью эквивариантных накрывающих функций (теорема 1) и расширенных эквивариантных накрывающих функций (теорема 2). Доказаны теоремы о связях $G$-расслоений с порожденными ими расслоениями (теоремы $3,4,5$ и следствия 1,2 ). Исследуются локальные и глобальные свойства $G$-расслоений. Доказан эквивариантный аналог теоремы Гуревича об эквивалентности понятий локального и глобального $G$-расслоения в случае паракомпактной базы (теорема 7). Определяется понятие слабо локально тривиального $G$-расслоения (определение 3$)$. Класс этих расслоений шире класса локально тривиальных $G$-расслоений, но в случае паракомпактной базы у́же класса $G$-расслоений Гуревича (теорема 8). При некотором дополнительном ограничении на базу расслоения классы слабо локально тривиальных $G$-расслоений и $G$ расслоений Гуревича совпадают (теорема 10). Дана классификация $G$-расслоений со свойством единственности накрывающего пути (теорема 11).

Используемые в статье без ссылок понятия, определения и результаты из эквивариантной топологии и теории расслоений можно найти в $[2,3,5,7]$.

2. $G$-Расслоения и их внутренняя характеристика. Пусть $G$-компактная топологическая группа, $E$ и $B-G$-пространства, а $p: E \rightarrow B$ - эквивариантное отображение.

Действие группы $G$ на $B$ индуцирует естественное действие группы $G$ на пространстве путей $B^{I}$ формулой $(g \alpha)(t)=g \alpha(t)$ для произвольных $g \in G, \alpha \in B^{I}, t \in I$. Рассмотрим подпространство $\Delta \subset E \times B^{I}$, определенное формулой

$$
\Delta=\left\{(e, \alpha) \in E \times B^{I} ; \alpha(0)=p(e)\right\} .
$$

Очевидно, что $\Delta$ - инвариантное подмножество $G$-пространства $E \times B^{I}$.

Определение 1. Эквивариантное отображение $\lambda: \Delta \rightarrow E^{I}$, удовлетворяющее условиям

$$
\lambda(e, \alpha)(0)=e, \quad[p \circ \lambda(e, \alpha)](t)=\alpha(t),
$$

называется эквивариантной накрывающей функиией или накрывающей $G$-функцией для $p$.

Следующая теорема описывает связь между эквивариантными расслоениями Гуревича и эквивариантными накрывающими функциями. Она дает внутреннюю характеристику эквивариантного расслоения Гуревича.

Теорема 1. Эквивариантное отображение $p: E \rightarrow B$ является $G$-расслоением Гуревича тогда и только тогда, когда р обладает эквивариантной накрывающей функи,ией.

Доказательство. Пусть $\lambda: \Delta \rightarrow E^{I}$ - эквивариантная накрывающая функция для $p$. Рассмотрим произвольные $G$-пространство $X$, эквивариантное отображение $\tilde{f}: X \times 0 \rightarrow E$ и эквивариантная гомотопия $F: X \times I \rightarrow B, F(x, 0)=p(\tilde{f}(x, 0))$. Заметим, что для произвольного $x \in X$ формулой $F_{x}(t)=F(x, t)$ определяется путь $F_{x} \in B^{I}$. Несложно убедиться, что гомотопия $\tilde{F}: X \times I \rightarrow E$, определенная формулой $\tilde{F}(x, t)=\lambda\left(\tilde{f}(x, 0), F_{x}\right)(t)$, является искомой эквивариантной накрывающей гомотопией.

Обратно, предположим, что $p: E \rightarrow B$ является $G$-расслоением Гуревича. Пусть $X=\Delta$; рассмотрим отображения

$$
\tilde{f}: \Delta \times 0 \rightarrow E, \quad \tilde{f}[(e, \alpha), 0]=e, \quad F: \Delta \times I \rightarrow B, \quad F[(e, \alpha), t]=\alpha(t) .
$$


Несложно проверить, что $\tilde{f}$ и $F$ являются эквивариантными отображениями и

$$
F[(e, \alpha), 0]=\alpha(0)=p(e)=(p \circ \tilde{f})[(e, \alpha), 0] .
$$

Следовательно, существует эквивариантная накрывающая гомотопия $\tilde{F}: \Delta \times I \rightarrow E$ для гомотопии $F$ :

$$
\tilde{F}[(e, \alpha), 0]=\tilde{f}, \quad p \circ \tilde{F}=F .
$$

Теперь определим отображение

$$
\lambda: \Delta \rightarrow E^{I}, \quad \lambda(e, \alpha)(t)=\tilde{F}[(e, \alpha), t] .
$$

Несложно заметить, что $\lambda$-эквивариантная накрывающая функция для $p$.

Пусть $W \subset B^{I}$ - инвариантное подмножество $G$-пространства $B^{I}$. Рассмотрим множество $\Delta_{W} \subset E \times B^{I} \times I$, определенное формулой

$$
\Delta_{W}=\{(e, \alpha, s) \in E \times W \times I ; \quad \alpha(s)=p(e)\} .
$$

Очевидно, $\Delta_{W}$ - инвариантное подмножество $G$-пространства $E \times B^{I} \times I$.

Определение 2. Эквивариантное отображение $\Lambda: \Delta_{W} \rightarrow E^{I}$, удовлетворяющее условиям

$$
\Lambda(e, \alpha, s)(s)=e, \quad[p \circ \Lambda(e, \alpha, s)](t)=\alpha(t),
$$

называется расширенной эквивариантной накрывающей функцией или расширенной накрывающей $G$-функиией над $W$.

Как показывает следующая лемма, существование расширенной накрывающей $G$-функции над $B^{I}$ эквивалентно существованию эквивариантной накрывающей функции.

Лемма 1. Эквивариантное отображение $p: E \rightarrow B$ обладает эквивариантной накрывающей функцией тогда и только тогда, когда существует расширенная накрывающая $G$-функиия над $B^{I}$.

Эта лемма доказывается так же, как аналогичное утверждение в неэквивариантном случае (см. [2, лемма 9, с. 124]).

Из теоремы 1 и леммы 1 непосредственно получается следующее утверждение.

Теорема 2. Эквивариантное отображение $p: E \rightarrow B$ является $G$-расслоением Гуревича тогда и только тогда, когда существует расширенная накрывающая $G$-бункиия над $B^{I}$.

Теоремы 1 и 2 имеют важное практическое значение и в дальнейшем неоднократно будут применены для доказательства того, что данное эквивариантное отображение является $G$-расслоением Гуревича.

3. $G$-Расслоения и порожденные ими расслоения. Пусть $H$ - замкнутая подгруппа компактной группы $G$. Тогда любое $G$-пространство является также $H$-пространством и любое $G$ отображение является $H$-отображением. Итак, существует естественный ковариантный функтор из категории $G$-ТОР в категорию $H$-ТОР. Возникает естественный вопрос: сохраняет ли этот функтор свойство эквивариантного отображения быть эквивариантным расслоением Гуревича. Ответ на этот вопрос дает следующая теорема.

Теорема 3. Пусть G-отображение $p: E \rightarrow B$ является $G$-расслоением Гуревича. Тогда для произвольной замкнутой подгруппь $H$ компактной группы $G$ отображение $p: E \rightarrow B$ является Н-расслоением Гуревича.

Доказательство. В силу теоремы 1 существует накрывающая $G$-функция $\lambda: \Delta \rightarrow E^{I}$ для $G$ отображения $p$. Так как $\lambda: \Delta \rightarrow E^{I}$ является также $H$-отображением, то $\lambda$ - накрывающая $H$ функция для $H$-отображения $p$. Значит, $p: E \rightarrow B$ является $H$-расслоение Гуревича в силу теоремы 1.

Из теоремы 3, в частности, вытекает следующее утверждение. 
Следствие 1. Произвольное $G$-расслоение Гуревича $p: E \rightarrow B$ является расслоением Гуревича.

Пусть $X$ и $Y-G$-пространства, а $H$ - некоторая замкнутая подгруппа группы $G$. Множество

$$
X^{H}=\{x \in X ; h x=x, \text { для всех } h \in H\}
$$

называется пространством $H$-неподвижсных точек $G$-пространства $X$. Эквивариантное отображение $f: X \rightarrow Y$ порождает естественное отображение между пространствами $H$-неподвижных точек $X^{H}$ и $Y^{H}$, которое обозначается через $p^{H}$.

Теорема 4. Пусть $p: E \rightarrow B$ является G-расслоением Гуревича. Тогда для произвольной замкнутой подгруппы $H$ компактной группы $G$ индуцированное отображение $p^{H}: E^{H} \rightarrow B^{H}$ между пространствами $H$-неподвижных точек является расслоением Гуревича.

Доказательство. В силу теоремы 1 для $G$-отображения $p$ существует накрывающая $G$-функция $\lambda: \Delta \rightarrow E^{I}$. Тогда $\lambda^{H}: \Delta^{H} \rightarrow\left(E^{H}\right)^{I}$ является накрывающей функцией для отображения $p^{H}:$ $E^{H} \rightarrow B^{H}$. Значит, $p^{H}: E^{H} \rightarrow B^{H}$ является расслоением Гуревича.

В случае, когда $G$-компактная группа Ли, а $E$ и $B-G$-CW-комплексы, верно и обратное утверждение теоремы 4 (см. [1, теорема 9]).

Свойство эквивариантного отображения быть $G$-расслоением Гуревича сохраняется также при переходе к пространствам $H$-орбит.

Теорема 5. Пусть $p: E \rightarrow B$ является $G$-расслоением Гуревича. Тогда для произвольной замкнутой подгруппы $H$ компактной группъ $G$ индуиированное $G$-отображение $p^{*}: E \mid H \rightarrow$ $B \mid H$ между пространствами $H$-орбит является $G$-расслоением Гуревича.

Доказательство. Пусть $\lambda: \Delta \rightarrow E^{I}$ - накрывающая $G$-функция для $G$-отображения $p$ (см. теорему 1). Рассмотрим множество

$$
\Delta_{H}=\left\{\left(e^{*}, \alpha^{*}\right) \in E \mid H \times(B \mid H)^{I} ; \alpha^{*}(0)=p^{*}\left(e^{*}\right)\right\}
$$

и определим отображение

$$
\lambda^{*}: \Delta_{H} \rightarrow(E \mid H)^{I}, \quad \lambda^{*}\left(e^{*}, \alpha^{*}\right)(t)=(\lambda(e, \alpha)(t))^{*} .
$$

Отображение $\lambda^{*}$ определено корректно и является накрывающей $G$-функцией для $p^{*}: E \mid H \rightarrow$ $B \mid H$. Следовательно, согласно теореме $1, p^{*}: E|H \rightarrow B| H$ является $G$-расслоением Гуревича.

Из последней теоремы в частном случае $H=G$ получается следующее утверждение.

Следствие 2. Пусть $p: E \rightarrow B$ является $G$-расслоением Гуревича. Тогда индуцированное отображение между пространствами орбит $p^{*}: E|G \rightarrow B| G$ является расслоением Гуревича.

4. Локальные и глобальные $G$-расслоения. Эквивариантное отображение $p: E \rightarrow B$ называется локальным $G$-расслоением Гуревича, если для произвольной точки $b \in B$ существует такая ее инвариантная окрестность $U$, что $\left.p\right|_{p^{-1}(U)}: p^{-1}(U) \rightarrow U$ является $G$-расслоением Гуревича.

Открытое покрытие $\mathcal{U}=\left\{U_{i} ; i \in \mathcal{I}\right\} G$-пространства $X$, состоящее из инвариантных подмножеств $U_{i}$, называется $G$-покрытием.

$G$-Покрытие $\mathcal{U}=\left\{U_{i} ; i \in \mathcal{I}\right\}$ называется нормальным $G$-покрытием, если для каждого $U_{i}$ существует такая функция $\chi_{i}: X \rightarrow I$, что $\chi_{i}(g x)=\chi_{i}(x)$ для произвольных $x \in X$ и $g \in G$, а $U_{i}=\left\{x \in X ; \chi_{i}(x) \neq 0\right\}$. Функция $\chi_{i}$ называется характеристической $G$-функцией открытого инвариантного подмножества $U_{i} \subset X$.

Лемма 2. Пусть $G$ - компактная группа. Тогда произвольное паракомпактное G-пространство $X$ обладает локально конечным нормальным $G$-покрытием.

Доказательство. Пусть $\mathcal{U}=\left\{U_{i} ; i \in \mathcal{I}\right\}$ - локально конечное нормальное покрытие, состоящее из открытых инвариантных подмножеств паракомпактного $G$-пространства $X$, а $h_{i}: X \rightarrow I$-характеристическая функция (не эквивариантная) подмножества $U_{i}, i \in \mathcal{I}$. Тогда функция $\chi_{i}: X \rightarrow I$, 
определенная с помощью интеграла Хаара формулой $\chi_{i}(x)=\int h_{i}(g x) d g$, является характеристической $G$-функцией инвариантного подмножества $U_{i}$. Значит, $\mathcal{U}=\left\{U_{i} ; i \in \mathcal{I}\right\}$ 一локально конечное нормальное $G$-покрытие паракомпактного $G$-пространства $X$.

Следующая теорема показывает, что при достаточно общих предположениях локальное $G$ расслоение Гуревича является глобальным $G$-расслоением Гуревича.

Теорема 6. Пусть $G$-компактная группа, $p: E \rightarrow B$-эквивариантное отображение, a $\mathcal{U}=\left\{U_{i} ; i \in \mathcal{I}\right\}$-локально конечное нормальное $G$-покрытие $G$-пространства $B$. Если $\left.p\right|_{p^{-1}\left(U_{i}\right)}: p^{-1}\left(U_{i}\right) \rightarrow U_{i}$ является $G$-расслоением Гуревича для произвольного $U_{i} \in \mathcal{U}$, то $p-$ G-расслоение Гуревича.

Доказательство. Для всякого конечного набора индексов $i_{1}, \ldots, i_{k} \in \mathcal{I}(k \geqslant 1)$ определим множество $V_{i_{1} \ldots i_{k}} \subset B^{I}$ следующим образом:

$$
V_{i_{1} \ldots i_{k}}=\left\{\alpha \in B^{I} ; \quad \alpha\left(\left[\frac{j-1}{k}, \frac{j}{k}\right]\right) \subset U_{i_{j}}, \quad j=1, \ldots, k\right\} .
$$

Ясно, что $V_{i_{1} \ldots i_{k}}$ - инвариантное подмножество $G$-пространства $B^{I}$. Докажем, что существует расширенная накрывающая $G$-функция над каждым $V_{i_{1} \ldots i_{k}}$. В силу теоремы 4 существует расширенная накрывающая $G$-функция $\Lambda_{j}: \Delta_{U_{i_{j}}} \rightarrow E^{I}$ над каждым $U_{i_{j}}, j=1, \ldots, k$. Рассмотрим произвольный элемент $(e, \alpha, s) \in \Delta_{V_{i_{1} \ldots i_{k}}}$. Предположим, что $s \in[(n-1) / k, n / k]$ для некоторого натурального $n$. Пусть $\alpha_{j} \in B^{I}, j=1, \ldots, k,-$ путь, определенный формулой

$$
\alpha_{j}(t)= \begin{cases}\alpha\left(\frac{j-1}{k}\right), & t \in\left[0, \frac{j-1}{k}\right], \\ \alpha(t), & t \in\left[\frac{j-1}{k}, \frac{j}{k}\right], \\ \alpha\left(\frac{j}{k}\right), & t \in\left[\frac{j}{k}, 1\right] .\end{cases}
$$

Теперь определим расширенную накрывающую $G$-функцию $\Lambda: \Delta_{V_{i_{1} \ldots i_{k}}} \rightarrow E^{I}$ следующим образом. Путь $\Lambda(e, \alpha, s)$ сначала определим на отрезке $[(n-1) / k, n / k]$ формулой

$$
\Lambda(e, \alpha, s)(t)=\Lambda_{n}\left(e, \alpha_{n}, s\right), \quad t \in\left[\frac{n-1}{k}, \frac{n}{k}\right] .
$$

Теперь $\Lambda(e, \alpha, s)$ определим последовательно на отрезках $[(n-2) / k,(n-1) / k],[(n-3) / k,(n-2) / k]$, $\ldots,[0,1 / k]$ формулой

$$
\Lambda(e, \alpha, s)(t)=\Lambda_{j}\left(\Lambda(e, \alpha, s)\left(\frac{j}{k}\right), \alpha_{j}, \frac{j}{k}\right), \quad t \in\left[\frac{j-1}{k}, \frac{j}{k}\right], j=n-1, n-2, \ldots, 1 .
$$

Наконец, на отрезках $[n / k,(n+1) / k],[(n+1) / k,(n+2) / k], \ldots,[(k-1) / k, 1]$ определим $\Lambda(e, \alpha, s)$ последовательно по формуле

$$
\Lambda(e, \alpha, s)(t)=\Lambda_{j+1}\left(\Lambda(e, \alpha, s)\left(\frac{j}{k}\right), \alpha_{j+1}, \frac{j}{k}\right), \quad t \in\left[\frac{j}{k}, \frac{j+1}{k}\right], j=n, n+1, \ldots, k-1 .
$$

Очевидно, что построенное эквивариантное отображение $\Lambda: \Delta_{V_{i_{1} \ldots i_{k}}} \rightarrow E^{I}$ удовлетворяет определению 2 , т.е. является расширенной накрывающей $G$-функцией над $V_{i_{1} \ldots i_{k}}$.

Семейство $\left\{V_{i_{1} \ldots i_{k}} ; k=1,2, \ldots\right\}$ является нормальным $G$-покрытием $G$-пространства $B^{I}$ и обладает вписанным $G$-покрытием $\left\{W_{\mu}\right\}$ с линейно упорядоченным множеством индексов $\mathcal{M}$, которое является также локально конечным (см. [4, лемма 3.4] или [6]).

Пусть $\chi_{\mu}: B^{I} \rightarrow I$-характеристическая $G$-функция инвариантного подмножества $W_{\mu} \subset B^{I}$, т.е. $\chi_{\mu}(g \alpha)=\chi_{\mu}(\alpha)$ для произвольных $g \in G, \alpha \in B^{I}$, и $W_{\mu}=\left\{\alpha \in B^{I} ; \chi_{\mu}(\alpha) \neq 0\right\}$. Расширенную накрывающую $G$-функцию над инвариантным множеством $W_{\mu} \subset B^{I}$ обозначим через $\Lambda_{\mu}$. Теперь рассмотрим произвольный элемент $(e, \alpha) \in \Delta \subset E \times B^{I}$. Пусть $\mu_{1}<\mu_{2}<\ldots<\mu_{n}$ множество всех 
тех индексов для которых выполняется $\alpha \in W_{\mu_{i}}, i=1, \ldots, n$. Определим точки $t_{i}, i=1, \ldots, n$, единичного отрезка $I$ формулой

$$
t_{i}=\frac{\sum_{j=1}^{i} \chi_{\mu_{j}}(\alpha)}{\sum_{j=1}^{n} \chi_{\mu_{j}}(\alpha)}, \quad i=1, \ldots, n .
$$

Построим путь $\lambda(e, \alpha) \in E^{I}$, определив его последовательно на отрезках $\left[0, t_{1}\right],\left[t_{1}, t_{2}\right], \ldots,\left[t_{n-1}, 1\right]$ следующими формулами

$$
\begin{gathered}
\lambda(e, \alpha)(t)=\Lambda_{\mu_{1}}(e, \alpha, 0)(t), \quad t \in\left[0, t_{1}\right] \\
\lambda(e, \alpha)(t)=\Lambda_{\mu_{i+1}}\left(\lambda(e, \alpha)\left(t_{i}\right), \alpha, t_{i}\right)(t), \quad t \in\left[t_{i}, t_{i+1}\right], \quad i=1,2, \ldots, n-1 .
\end{gathered}
$$

Очевидно, что $\lambda(e, \alpha)(0)=e$ и $[p \circ \lambda(e, \alpha)](t)=\alpha(t)$. Кроме того, $\lambda: \Delta \rightarrow E^{I}$ - эквивариантное отображение, поскольку таковыми являются $\Lambda_{\mu}, \mu \in \mathcal{M}$. Значит, $\lambda: \Delta \rightarrow E^{I}$ - накрывающая $G$ функция для $p$, т.е. $p: E \rightarrow B-G$-расслоение Гуревича согласно теореме 1 .

Из последней теоремы и леммы 2 непосредственно вытекает следующая теорема.

Теорема 7. Пусть $G$-компактная группа, а $B$ - паракомпактное $G$-пространство. Эквивариантное отображение $p: E \rightarrow B$ является $G$-расслоением Гуревича тогда и только тогда, когда оно является локальным $G$-расслоением Гуревича.

Итак, в случае паракомпактной базы $B$ понятия локального и глобального $G$-расслоения совпадают. Теорема 7 является эквивариантным аналогом известной теоремы Гуревича (см. [6]).

5. Слабо локально тривиальные $G$-расслоения. Пусть $G$-компактная группа, $B$ и $F-$ произвольные $G$-пространства, а $p: B \times F \rightarrow B$ - проекция на первый сомножитель: $p(x, y)=x$. Эквивариантное отображение $p: B \times F \rightarrow B$ является $G$-расслоением Гуревича. Действительно, пусть $X$ - произвольное $G$-пространство, $\tilde{f}: X \rightarrow B \times F$-эквивариантное отображение, а $F$ : $X \times I \rightarrow B$ - такая эквивариантная гомотопия, что $F(x, 0)=p \tilde{f}(x), x \in X$. Очевидно, что отображение $\tilde{F}: X \times I \rightarrow B \times F$, определенное формулой $\tilde{F}(x, t)=\left(F(x, t), p r_{2}(\tilde{f}(x, 0))\right)$, является эквивариантной накрывающей гомотопией для $F: X \times I \rightarrow B$. Проекция $p: B \times F \rightarrow B$ называется тривиальным G-расслоением.

Эквивариантное отображение $p: E \rightarrow B$ называется локально тривиальным $G$-расслоени$e м$, если для произвольной точки $b \in B$ существует такая ее инвариантная окрестность $U$, что $\left.p\right|_{p^{-1}(U)}: p^{-1}(U) \rightarrow U$ является тривиальным $G$-расслоением.

Из теоремы 7 следует, что если $B$ паракомпактное $G$-пространство, то локально тривиальное $G$-расслоение $p: E \rightarrow B$ является $G$-расслоением Гуревича.

Определение 3. Эквивариантное отображение $p: E \rightarrow B$ назовем слабо локалъно тривиальным $G$-расслоением, если для произвольной точки $G$-пространства $B$ существуют открытая инвариантная окрестность $U$ этой точки и эквивариантное отображение $\omega: U \times p^{-1}(U) \rightarrow p^{-1}(U)$, что

(i) $p \circ \omega(b, e)=b$ для всех $(b, e) \in U \times p^{-1}(U)$,

(ii) $\omega(p(e), e)=e$ для всех $e \in p^{-1}(U)$.

Условие (i) последнего определения означает, что композиция

$$
U \times p^{-1}(U) \stackrel{\omega}{\longrightarrow} p^{-1}(U) \stackrel{p}{\longrightarrow} U
$$

представляет собой проекцию на первый сомножитель. Очевидно, что локально тривиальное $G$ расслоение $p: E \rightarrow B$ является слабо локально тривиальным $G$-расслоением. Но, в отличие от тривиального $G$-расслоения, эквивариантное отображение $\omega: U \times p^{-1}(U) \rightarrow p^{-1}(U)$ в определении 3 , вообще говоря, не является эквивариантным гомеоморфизмом.

Лемма 3. Слабо локально тривиальное $G$-расслоение $p: E \rightarrow B$ является локальным $G$ расслоением Гуревича. 
Доказательство. Пусть $b_{0} \in B$ - произвольная точка. Рассмотрим инвариантную окрестность $U$ этой точки и эквивариантное отображение $\omega: U \times p^{-1}(U) \rightarrow E$, которое удовлетворяет условиям (i) и (ii) определения 3. Докажем, что $\left.p\right|_{p^{-1}(U)}: p^{-1}(U) \rightarrow U$ является $G$-расслоением Гуревича. Определим отображение $\lambda: \Delta \rightarrow\left(p^{-1}(U)\right)^{I}$ формулой $\lambda(e, \alpha)(t)=\omega(\alpha(t), e)$. Несложно проверить, что $\lambda$ является накрывающей $G$-функцией для $G$-отображения $\left.p\right|_{p^{-1}(U)}$.

Из последней леммы, учитывая теорему 7 , непосредственно получаем следующее утверждение.

Теорема 8. Пусть $p: E \rightarrow B$ - слабо локально тривиальное $G$-расслоение, где $B$ - паракомпактное $G$-пространство. Тогда $p: E \rightarrow B$ является $G$-расслоением Гуревича.

Обратное утверждение верно при достаточно слабых ограничениях на $G$-пространство $B$ (см. теорему 10).

Определение 4. $G$-пространство $X$ назовем эквивариантно равномерно локально стягиваемым, если для произвольной точки $x_{0} \in X$ существует такая инвариантная окрестность $U$ этой точки и такое эквивариантное отображение $\sigma: U \times U \times I \rightarrow X$, что выполняются следующие условия:

(i) $\sigma(x, y, 0)=x$ и $\sigma(x, y, 1)=y$ для всех $(x, y) \in U \times U$,

(ii) $\sigma(x, x, t)=x$ для всех $x \in U$ и $t \in I$.

Как показывает следующая лемма, эквивариантно равномерно локально стягиваемых $G$-пространств много.

Лемма 4. Произвольное $G$-ANR-пространство является эквивариантно равномерно локально стягиваемым.

Доказательство этой леммы простое и оно оставляется читателю.

Из леммы 4 , в частности, следует, что $G$-CW-комплексы являются эквивариантно равномерно локально стягиваемыми $G$-пространствами.

Теорема 9. Пусть $B$-эквивариантно равномерно локально стягиваемое $G$-пространство. Тогда $p: E \rightarrow B$ является локальным $G$-расслоением Гуревича тогда и только тогда, когда $p: E \rightarrow B-$ слабо локально тривиальное $G$-расслоение.

Доказательство. Достаточность утверждения без дополнительных ограничений на $G$-пространство $B$ доказана в лемме 3 .

Докажем необходимость. Пусть $p: E \rightarrow B$-локальное $G$-расслоение Гуревича. Для произвольной точки $b_{0} \in B$ рассмотрим такую эквивариантную окрестность $V$ точки $b_{0}$, что $\left.p\right|_{p^{-1}(V)}: p^{-1}(V) \rightarrow V$ является $G$-расслоением Гуревича. Пусть $\lambda: \Delta \rightarrow\left(p^{-1}(V)\right)^{I}-$ накрывающая $G$-функция отображения $\left.p\right|_{p^{-1}(V)}$. Так как $B$ - эквивариантно равномерно локально стягиваемое $G$-пространство, то существуют такие инвариантная окрестность $U$ точки $b_{0}, U \subset V$, и эквивариантное отображение $\sigma: U \times U \times I \rightarrow B$, что выполняются условия $\sigma\left(b, b^{\prime}, 0\right)=b$, $\sigma\left(b, b^{\prime}, 1\right)=b^{\prime}$ и $\sigma(b, b, t)=b$ для всех $b, b^{\prime} \in U$ и $t \in I$. При этом инвариантную окрестность $U$ можно выбрать так, что $\sigma(U \times U \times I) \subset V$. Для этого достаточно заметить, что $\left(b_{0}, b_{0}, I\right) \in \sigma^{-1}(V)$ и применить компактность $I$.

Пусть теперь $(b, e) \in U \times p^{-1}(U)$. Рассмотрим путь $\tilde{\alpha} \in B^{I}$, определенный формулой $\tilde{\alpha}(t)=$ $\sigma(p(e), b, t)$. Заметим, что $\tilde{\alpha}$ соединяет точку $p(e)$ с точкой $b$. Определим эквивариантное отображение $\omega: U \times p^{-1}(U) \rightarrow p^{-1}(U)$ формулой

$$
\omega(b, e)=\lambda(e, \tilde{\alpha})(1) .
$$

Несложно убедиться, что $\omega$ удовлетворяет условиям (i) и (ii) определения 3.

Из теорем 7 и 9 непосредственно получается следующая важная для применения теорема.

Теорема 10. Пусть $B$ - паракомпактное и эквивариантно равномерно локально стягиваемое $G$-пространство. Тогда $p: E \rightarrow B$ является $G$-расслоением Гуревича тогда и только тогда, когда $p: E \rightarrow B$ - слабо локально тривиальное $G$-расслоение. 
6. $\quad G$-Расслоения со свойством единственности накрывающего пути. Говорят, что отображение $p: E \rightarrow B$ обладает свойством единственности накрывающего пути, если для любых двух путей $\alpha, \alpha^{\prime}: I \rightarrow E$, которые удовлетворяют условиям $\alpha(0)=\alpha^{\prime}(0)$ и $p \circ \alpha=p \circ \alpha^{\prime}$, выполняется равенство $\alpha=\alpha^{\prime}$.

Рассмотрим произвольные $G$-пространства $E$ и $B$, где $G$ - компактная топологическая группа.

Лемма 5. Пусть эквивариантное отображение $p: E \rightarrow B$ является расслоением Гуревича со свойством единственности накрывающего пути. Тогда $p: E \rightarrow B-G$-расслоение Гуревича.

Доказательство. Пусть $X$-произвольное $G$-пространство, а $\tilde{f}: X \rightarrow E$ и $F: X \times I \rightarrow B-$ произвольные эквивариантные отображения, удовлетворяющие условиям $F(x, 0)=(p \circ \tilde{f})(x)$. Так как $p: E \rightarrow B$ - расслоение Гуревича, то существует такая гомотопия $\tilde{F}: X \times I \rightarrow E$, что $\tilde{F}(x, 0)=\tilde{f}(x)$ и $p \circ \tilde{F}=F$. Для завершения доказательства достаточно показать, что накрывающая гомотопия $\tilde{F}$ на самом деле является эквивариантной накрывающей гомотопией. Рассмотрим пути $\alpha, \alpha^{\prime}: I \rightarrow E$, определенные формулами

$$
\alpha(t)=\tilde{F}(g x, t) \text { и } \alpha^{\prime}(t)=g \tilde{F}(x, t),
$$

где $g \in G, x \in X$ - произвольные фиксированные элементы. Заметим, что

$$
\alpha(0)=\tilde{F}(g x, 0)=\tilde{f}(g x)=g \tilde{f}(x), \quad \alpha^{\prime}(0)=g \tilde{F}(x, 0)=g \tilde{f}(x) .
$$

Итак, $\alpha(0)=\alpha^{\prime}(0)$. Кроме того,

$$
\begin{gathered}
(p \circ \alpha)(t)=(p \circ \tilde{F})(g x, t)=F(g x, t)=g F(x, t), \\
\left(p \circ \alpha^{\prime}\right)(t)=p(g \tilde{F}(x, t))=g p(\tilde{F}(x, t))=g(p \circ \tilde{F})(x, t)=g F(x, t) .
\end{gathered}
$$

Таким образом, $p \circ \alpha=p \circ \alpha^{\prime}$. Значит, $\alpha=\alpha^{\prime}$, т.е. $\tilde{F}(g x, t)=g \tilde{F}(x, t)$, поскольку $p: E \rightarrow B$ обладает свойством единственности накрывающего пути.

Из леммы 5 и следствия 1 непосредственно получается следующая теорема.

Теорема 11. Эквивариантное отображение $p: E \rightarrow B$ со свойством единственности накрывающего пути является $G$-расслоением Гуревича тогда и только тогда, когда оно является расслоением Гуревича.

Иначе говоря, в классе всех эквивариантных отображений со свойством единственности накрывающего пути понятия $G$-расслоения Гуревича и расслоения Гуревича совпадают.

\section{СПИСОК ЛИТЕРАТУРЫ}

1. Геворкян П. С., Хименес Р. Об эквивариантных расслоениях G-CW-комплексов// Мат. сб. - 2019. 210, № 10. - C. 91-98.

2. Спенъер Э. Алгебраическая топология. - М.: Мир, 1971.

3. tom Dieck T. Transformation Groups. - Walter de Gruyter, 1987.

4. Dugundji J. Topology. — Boston, Massachusetts: Allyn and Bacon, 1960.

5. James I. M., Segal G. B. On equivariant homotopy type// Topology. - 1978. — 17. — P. 267-272.

6. Hurewicz W. On the concept of fiber space// Proc. Natl. Acad. Sci. U.S.A. - 1955. - 41. - P. 956-961.

7. May J., Sigurdsson J. Parametrized Homotopy Theory. — Am. Math. Soc., 2006.

Геворкян Павел Самвелович

Московский педагогический государственный университет

E-mail: pgev@yandex.ru 